\title{
Paraoxonase 1 Gene Polymorphism in Patients with Atopic Dermatitis
} N.W.Mikhael ${ }^{1}$, S.A.Mohammed ${ }^{2}$, A.I.Mustafa ${ }^{1}$ and M.I.Elbadrawy ${ }^{1}$

${ }^{1}$ Dermatology, Venereology and Andrology Dept., Faculty of Medicine, Benha Univ., Benha, Egypt

${ }^{2}$ Medical Biochemistry and Molecular Biology Dept., Faculty of Medicine, Benha Univ., Benha, Egypt E-Mail: Elbadrawy56@gmail.com

\begin{abstract}
Atopic dermatitis, commonly called atopic eczema were considered to be a one sort of persistent and backsliding provocative sicknesses of skin with high heterogeneity and heritability. The principle point of this examination was to research the relationship between Paroxonase 1 quality 192Q/R and 55L/M polymorphisms and atopic dermatitis. Subjects and techniques: This was an imminent case-control study included 100 patients experiencing atopic dermatitis (AD) (Group A). Notwithstanding 100 obviously solid people of coordinated age and sex as a benchmark (Group B). Discovery of single nucleotide polymorphism (SNP) for paroxonase 1 quality $(192 \mathrm{Q} / \mathrm{R}$ and $55 \mathrm{~L} / \mathrm{M})$ was finished utilizing explicit preliminaries by a polymerase chain response - based limitation section length polymorphism (PCRRFLP) test .Results: L allele demonstrated fundamentally higher recurrence in AD patients when contrasted with control gatherings; with danger to grow AD. While 192Q/R RR, RQ+RR genotypes, R allele; 55L/M MM, ML+MM genotypes, $M$ allele had lower recurrence in $\mathrm{AD}$ patients when contrasted with control gatherings. End: The QL haplotype was altogether connected with danger of AD. Further examination utilizing the rules and all the more fittingly fueled investigations ought to incredibly improve the trust in the gauge of danger factors and an impact of a specific way to deal with treatment.
\end{abstract}

Keywords: Paraoxonase 1 Gene Polymorphism, Atopic Dermatitis

\section{Introduction}

Atopic dermatitis (AD) is a typical provocative skin infection influencing up to $25 \%$ of kids and up to $10 \%$ of grown-ups in Western industrialized nations. The illness is clinically described by intensifications and reductions of eczematous skin with aggravation, pruritus and abrasions, scaling, dry skin, and weakness for cutaneous bacterial and mycotic diseases [1].

The pathogenesis of $\mathrm{AD}$ is perplexing and multifactorial. Skin obstruction brokenness, ecological elements, hereditary inclination, and invulnerable brokenness all assume a function in its turn of events and are firmly entwined [2].

Oxidative pressure has likewise been ensnared in the pathogenesis of $\mathrm{AD}$. It's characterized as the development of oxidants in the phones of the human body that intensely or persistently surpasses the cell reinforcement protection limit. Oxidants, including free revolutionaries, responsive oxygen species (ROS), and nitrogen oxygen species (NOS) and receptive metabolites are delivered during typical metabolic exercises. It is notable that oxidative pressure advances tissue aggravation through up guideline of qualities that code supportive of fiery cytokines [3].

Human serum paraoxonase-1 (PON1) shows two polymorphisms in the coding area (Q192R and L55M) and a few polymorphisms in the advertiser areas. The Q192R polymorphism influences reactant efficiency, while the L55M polymorphism influences catalyst fixation [4].

Serum paraoxonase-1 (PON1) is a calciumsubordinate esterase/lactonase, which is and principally incorporated by the liver and flows inside HDL particles. PON1 has PON, aryl esterase, and lactonase exercises. The catalyst not just hydrolyzes a few organ phosphorus bug sprays and nerve specialists, which is engaged with the insurance against xenobiotic harmfulness, has cell reinforcement, calming, and hostile to atherogenic properties [5].

The point of this examination was to research the relationship between Paroxonase 1 quality 192Q/R and $55 \mathrm{~L} / \mathrm{M}$ polymorphisms and atopic dermatitis.

\section{Patient and method}

This was a planned case-control study included 100 patients experiencing atopic dermatitis (AD) (Group A). Notwithstanding 100 clearly sound people of coordinated age and sex as a benchmark (Group B).

\section{Consideration measures}

Persistent with $\mathrm{AD}$ and conclusion depended on clinical discoveries as indicated by the example of American Academy of Dermatology agreement meeting on pediatric atopic dermatitis (Echenfield et al., 2014).

Patients with contrast levels of AD seriousness According to SCORE AD(E Rusu et al.,2019).

\section{Prohibition measures}

Any subject avoided from the investigation on the off chance that he/she was:

- Patients with history of inborn coronary illness.

- Patients with history of uncontrolled diabetes mellitus.

- Patient with history of asthma at season of study.

- Patients with history of renal disappointment.

- Patients with history of urticaria.

All patients were exposed to finish general and dermatological clinical assessment to clinically evaluate atopic sores, decide the circulation and the degree of $\mathrm{AD}$. Clinical information of all patients are recorded 
Recognition of single nucleotide polymorphism (SNP) for paroxonase 1 quality (192 Q/R and $55 \mathrm{~L} / \mathrm{M})$ was finished utilizing explicit preliminaries by a polymerase chain response - based limitation part length polymorphism (PCR-RFLP) assay

\section{Results}

The paroxenase $1 \quad$ (PON1) 192 Q/R, 55L/M haplotypes were calculated; RM showed the highest prevalence in control, as well as in patients; while RL showed the lowest prevalence in control, and AD groups.

The QL haplotype was significantly associated with risk of $\mathrm{AD}$ occurrence $(\mathrm{p}=0.022, \mathrm{OR}=2.228)$.

No significant differences were found in demographic data, FH, BMI, and associated conditions between $192 \mathrm{Q} / \mathrm{R}$ genotypes in all studied AD patients

SCORAD score increased gradually between RR, RQ, QQ respectively $(\mathrm{p}<0.001)$, dominant model (RQ+QQ) showed significantly higher SCORAD score when compared to RR genotypes. Otherwise, no significant differences were found in $\mathrm{AD}$ features between $192 \mathrm{Q} / \mathrm{R}$ genotypes in all studied patients.

No significant differences were found in demographic data, FH, BMI, and associated conditions between 55L/M genotypes in all studied AD patients.

SCORAD score increased gradually between MM, ML, LL respectively, ML+LL showed significantly higher SCORAD score when compared to MM genotypes. Otherwise, no significant differences were found in $\mathrm{AD}$ features between 55L/M genotypes in all studied AD patients.

Regression analysis was conducted for prediction of AD occurrence, using age, gender, FH, BMI, PON1 genotypes as covariates. $192 \mathrm{Q} / \mathrm{R}$ (RQ+QQ), 55L/M (ML+LL) were significantly associated with risk of AD development, in uni- and multivariable analyses.

Regression analysis was conducted for prediction of higher SCORAD score in AD patients, using age, gender, FH, BMI, duration, onset, PON1 genotypes as covariates. $192 \mathrm{Q} / \mathrm{R}(\mathrm{RQ}+\mathrm{QQ})$, 55L/M (ML+LL) were associated with prediction of higher SCORAD score in uni- and multivariable analyses.

Table (1) Regression analysis for prediction of higher AD SCORAD score.

\begin{tabular}{|c|c|c|c|c|c|c|c|c|}
\hline \multirow{3}{*}{$\begin{array}{l}\text { Variable } \\
\text { Age }\end{array}$} & \multicolumn{4}{|c|}{ Univariable } & \multicolumn{4}{|c|}{ Multivariable } \\
\hline & \multirow{2}{*}{$\begin{array}{c}\mathbf{p} \\
0.313\end{array}$} & \multirow{2}{*}{$\begin{array}{c}\text { OR } \\
0.682\end{array}$} & \multicolumn{2}{|c|}{$95 \%$ CI } & \multirow[t]{2}{*}{$\mathbf{p}$} & \multirow[t]{2}{*}{ OR } & \multicolumn{2}{|c|}{$95 \% \mathrm{CI}$} \\
\hline & & & 0.543 & 1.750 & & & & \\
\hline Sex & 0.277 & 0.316 & 0.025 & 4.165 & & & & \\
\hline Positive FH & 0.325 & 2.941 & 0.075 & 7.740 & & & & \\
\hline BMI & 0.197 & 0.795 & 0.578 & 1.092 & & & & \\
\hline Duration & 0.177 & 0.475 & 0.467 & 1.177 & & & & \\
\hline Onset & 0.273 & 0.365 & 0.192 & 1.387 & & & & \\
\hline $\begin{array}{l}192 Q / R \\
(R Q+Q Q)\end{array}$ & $<0.04$ & 1.041 & 1.086 & 1.911 & $<0.03$ & 0.904 & 0.859 & 1.167 \\
\hline $\begin{array}{l}\text { R55L/M } \\
(M L+L L)\end{array}$ & $<0.03$ & 0.993 & 1.127 & 1.789 & $<0.03$ & 0.996 & 1.259 & 1.049 \\
\hline
\end{tabular}

OR, odds ratio; $\mathrm{CI}$, confidence interval.

\section{Discussion}

Human serum paraoxonase 1 (PON1) is an esterase chemical which partakes in the end of ROS by authoritative to high-thickness lipoprotein (HDL) and has profoundly lipophilic cancer prevention agent qualities. Notwithstanding the preventive part of PON1 against OS, which is thought to add to carcinogenesis, PON1 additionally adds to the detoxification of organophosphate mixes and cancer-causing lipid dissolvable ROS which are created by lipid peroxidation [6].

The current investigation demonstrated that no huge contrasts were found in segment information, $\mathrm{FH}$, BMI, and related conditions between $192 \mathrm{Q} / \mathrm{R}$ genotypes in totally concentrated $\mathrm{AD}$ patients.
SCORAD score expanded step by step between RR, RQ, QQ individually $(\mathrm{p}<0.001)$, predominant model (RQ+QQ) demonstrated fundamentally higher SCORAD score when contrasted with RR genotypes. Something else, no huge contrasts were found in AD includes between 192 Q/R genotypes in completely examined patients. No critical contrasts were found in segment information, FH, BMI, and related conditions between 55L/M genotypes in completely concentrated AD patients. SCORAD score expanded bit by bit between MM, ML, LL separately ( $\mathrm{p}<0.001)$, (ML+LL) demonstrated fundamentally higher SCORAD score when contrasted with MM genotypes. Something else, no critical contrasts were found in $\mathrm{AD}$ includes 
between 55L/M genotypes in completely concentrated $\mathrm{AD}$ patients.

Atopic dermatitis (AD), unfavorably susceptible rhinitis, and asthma establish the ternion of atopic diseases.The SCORAD (Index) is the best approved scoring framework in atopic dermatitis (AD). Alteration of the SCORAD Index has driven a few times to mistaken utilization of the framework. To quantify the degree of $\mathrm{AD}$, the standard of nines is applied on a front/back drawing of the patient's fiery injuries [7].

Celakovská and Bukač, [8] showed atopic walk in patients with $\mathrm{AD}$ on the grounds that the event of bronchial asthma, unfavorably susceptible rhinitis, and tenacious eczematous injuries is fundamentally higher in moderate and extreme types of $\mathrm{AD}$ assessed with the SCORAD list. The altogether rising event of bronchial asthma in mellow, moderate, and extreme types of $\mathrm{AD}$ was $35 \%, 45 \%$, and $64 \%$, individually. The altogether rising event of unfavorably susceptible rhinitis in mellow, moderate, and extreme types of AD was $65 \%$, $76 \%$, and $96 \%$, individually. The essentially rising event of tenacious eczematous injuries in mellow, moderate, and extreme types of $\mathrm{AD}$ was $38 \%, 69 \%$, and $96 \%$, separately.

The current investigation indicated that relapse examination was led for expectation of $\mathrm{AD}$ event, utilizing age, sexual orientation, FH, BMI, PON1 genotypes as covariates. $192 \mathrm{Q} / \mathrm{R}(\mathrm{RQ}+\mathrm{QQ}), 55 \mathrm{~L} / \mathrm{M}$ (ML+LL) were fundamentally connected with danger of $\mathrm{AD}$ advancement, in uni-and multivariable examinations. Relapse investigation was led for expectation of higher SCORAD score in AD patients, utilizing age, sex, FH, BMI, term, beginning, PON1 genotypes as covariates. $192 \mathrm{Q} / \mathrm{R}(\mathrm{RQ}+\mathrm{QQ}), 55 \mathrm{~L} / \mathrm{M}$ (ML+LL) were related with expectation of higher SCORAD score in uni-and multivariable examinations.

Of the 3 PONs, PON1 has been most seriously concentrated comparable to the danger of oxidative pressure and irritation. Its encoding quality has additionally been recognized as a life span quality . PON1 is integrated in the liver and flows on the outside of HDL particles, and it is a Ca2+-connected catalyst. It was first read for its organophosphates action which disclosed its capacity to detoxify organophosphate through hydrolysis and consequently give neuroprotection against the impacts of natural neurotoxins and age-related neurodegeneration. Hence, PON1 was attributed to have critical enemy of oxidative and mitigating properties through its enzymatic activities as a lactonase, peroxidase and esterase. These properties represent the capacity of HDL to forestall LDL oxidation [9].

Paraoxonase 1 has additionally been demonstrated to be associated with arylesterase (ARE), lactonase, phospholipase A2-like movement, estrogen-ester hydrolase, cyclic carbamate hydrolase and conceivably peroxidase-like action. PON1 can catalyze the hydrolysis of a few exogenous and endogenous mixes; a particular endogenous substrate has not been found.
Modifications in flowing PON1 levels and action have been accounted for in a few illnesses including oxidative pressure and aggravation [10].

Paraoxonase 1 is available in other immune system issues. In the investigation of Collazo et al., [11], the presence of the PON1 $55 \mathrm{M}$ allele wasn't discovered to be related with psoriasis; yet PON1 $192 \mathrm{R}$ allele was discovered to be related with psoriasis in their populace. Arora et al., 2015 discovered critical diminished degrees of serum PON-1 in psoriatic patients when contrasted with control gathering.

Ramadan et al., [12] exhibited that all patients with vitiligo and alopecia areata (AA) indicated altogether lower levels of PON1 in tissue and serum than the controls $(\mathrm{p}<0.001)$.

Takci et al., [13] uncovered that in rosacea bunch mean serum paraoxonase and arylesterase exercises were $74.54 \pm 38.30 \mathrm{U} \mathrm{L}-1$ and $141.29 \pm 22.27 \mathrm{kU} \mathrm{L}-1$ separately, which were fundamentally lower than controls $(\mathrm{P}=0.010,0.005$; individually).

In an investigation led by El-Lebedy et al., [14], they uncovered that PON1 192R and $55 \mathrm{~L}$ alleles are related with T2DM. Q192R polymorphism is related with CVD and lower serum compound focus and may speaks to a novel danger factor for CVD in Egyptian patients with T2DM. Human serum paraoxonase-1 (PON-1) is a compound combined in the liver and delivered in blood official to HDL. It is a cell reinforcement catalyst that hydrolyzes the poisonous peroxides in the oxidized lipids in both LDL and HDL; subsequently, it has been involved in the anticipation of atherosclerosis. Diminished PON-1 movement, free of genotype, has been reported in diabetes, hypercholesterolemia. It was noticed that patients with low PON-1 action are more defenseless against sicknesses including expanded oxidative harm and lipid peroxidation contrasted and patients with high PON-1 action [15].

In view of our discoveries, we suggest for additional investigations on huge example size and huge geological scale to underscore our decision. Planning and actualizing instructive projects (wellbeing schooling) that concerning the atopic dermatitis and its danger factors and related illnesses including the solid nourishment and way of life, practice and different perspectives that influence the whole health.

\section{References}

[1] B.Cabanillas, A.C.Brehler, N.Novak. Atopic dermatitis phenotypes and the need for personalized medicine. Current Opinion in Allergy and Clinical Immunology, Vol.17(4), PP.309,2017.

[2] P.M.Elias, M.Steinhoff. "Outside-to-inside"(and now back to "outside") pathogenic mechanisms in atopic dermatitis. J.,Investigative Dermatology, Vol.128(5), PP.1067-1070,2008.

[3] H.Ji, X.K.Li. Oxidative stress in atopic dermatitis. Oxid Med Cell Longev, Vol.2016, PP.2721469,2016. 
[4] N.Ponce-Ruiz, F.E.Murillo-González, A.E.RojasGarcía. Transcriptional regulation of human paraoxonase 1 by nuclear receptors. Chemicobiological interactions, Vol.268, PP.77-84,2017.

[5] Y.Wang, H.Liu, P.Fan. Evidence for association between paraoxonase 1 gene polymorphisms and polycystic ovarian syndrome in south-west Chinese women. European J.,endocrinology, Vol.166(5), PP.877,2012.

[6] M.Eroglu, N.Yilmaz, S.Yalcinkaya. Enhanced HDL-cholesterol-associated anti-oxidant PON-1 activity in prostate cancer patients. Kaohsiung $\mathbf{J}$ Med Sci. Jul, Vol.29(7), PP.368-73,2013 .

[7] A.P.Oranje. Practical issues on interpretation of scoring atopic dermatitis: SCORAD Index, objective SCORAD, patient-oriented SCORAD and Three-Item Severity score. In Pathogenesis and Management of Atopic Dermatitis , Vol.41, pp. 149-155,2011.

[8] T.Celakovská, J.Bukač. The severity of atopic dermatitis evaluated with the SCORAD index and the occurrence of bronchial asthma and rhinitis, and the duration of atopic dermatitis. Allergy \& Rhinology, Vol.7(1), PP.ar,2016.

[9] L.P.Précourt, D.Amre, M.C.Denis. The three-gene paraoxonase family: physiologic roles, actions and regulation. Atherosclerosis, Vol.214, PP.2036,2011 .
[10]P.Huen, K.Yousefi, B.Street. PON1 as a model for integration of genetic, epigenetic, and expression data on candidate susceptibility genes, Environ. Epigenet. 1 pii: dvv003,2015.

[11] A.A.H. Collazo, A.A.Navarro, M.F.Morris. 475 Paraoxonase 1 (PON1) L55M and Q192R polymorphisms, lipid profiles and psoriasis. J.,Investigative Dermatology, Vol.137(5), PP.S82,2017.

[12] R.Ramadan, A.Tawdy, R.A.Hay. The antioxidant role of paraoxonase 1 and vitamin $\mathrm{E}$ in three autoimmune diseases. Skin pharmacology and physiology, Vol.26(1), PP.2-7,2013.

[13]Z.Takci, S.G.Bilgili, A.S.Karadag. Decreased serum paraoxonase and arylesterase activities in patients with rosacea. J.,the European Academy of Dermatology and Venereology, Vol.29(2), PP.367370,2015 .

[14]D.El-Lebedy, M.Kafoury, D.Abd-El Haleem. Paraoxonase-1 gene Q192R and L55M polymorphisms and risk of cardiovascular disease in Egyptian patients with type 2 diabetes mellitus. J.,Diabetes \& Metabolic Disorders, Vol.13(1), PP.124,2014.

[15]I.Seres, G.Paragh, E.Deschene. Study of factors influencing the decreased HDL associated PON1 activity with aging. Exp Gerontol, Vol.39, PP.5966,2004. 